\title{
Magnetic Properties and Biological Activity Evaluation of Iron Oxide Nanoparticles
}

\author{
Alina Mihaela Prodan, ${ }^{1,2}$ Simona Liliana Iconaru, ${ }^{3,4,5}$ Carmen Mariana Chifiriuc, ${ }^{6}$ \\ Coralia Bleotu, ${ }^{7}$ Carmen Steluta Ciobanu, ${ }^{3}$ Mikael Motelica-Heino, ${ }^{5}$ \\ Stanislas Sizaret, ${ }^{5}$ and Daniela Predoi ${ }^{3}$ \\ ${ }^{1}$ Carol Davila University of Medicine and Pharmacy, 8 Eroii Sanitari, Sector 5, Bucharest, Romania \\ ${ }^{2}$ Emergency Hospital Floreasca, Bucharest 5, Calea Floreasca Nr 8, Sector 1, Bucharest, Romania \\ ${ }^{3}$ National Institute of Materials Physics, 105 bis Atomistilor, P.O. Box MG 07, 077125 Bucuresti-Magurele, Romania \\ ${ }^{4}$ Faculty of Physics, University of Bucharest, 405 Atomistilor, CP MG-1, 077125 Magurele, Romania \\ ${ }^{5}$ ISTO, Université d'Orléans, Orléans Cedex 02, 45067, France \\ ${ }^{6}$ Microbiology Department, Faculty of Biology, University of Bucharest, Aleea Portocalelor 1-3, 60101 Bucharest, Romania \\ ${ }^{7}$ Institute of Virology, Antiviral Therapy Department "Stefan S. Nicolau", 285 Mihai Bravu, 030304 Bucharest, Romania
}

Correspondence should be addressed to Daniela Predoi; dpredoi@gmail.com

Received 14 March 2013; Revised 7 June 2013; Accepted 8 June 2013

Academic Editor: Anjan Barman

Copyright (C) 2013 Alina Mihaela Prodan et al. This is an open access article distributed under the Creative Commons Attribution License, which permits unrestricted use, distribution, and reproduction in any medium, provided the original work is properly cited.

\begin{abstract}
The aim of this study was to provide information about the biological properties of iron oxide nanoparticles (IO-NPs) obtained in an aqueous suspension. The IO-NPs were characterized by transmission electron microscopy (TEM). Analysis of hysteresis loops data at room temperature for magnetic IO-NPs sample indicated that the IO-NPs were superparamagnetic at room temperature. The calculated saturation magnetization for magnetic iron oxide was $\mathrm{M}_{\mathrm{s}}=18.1 \mathrm{emu} / \mathrm{g}$. The antimicrobial activity of the obtained PMC-NPs was tested against Gram-negative (Pseudomonas aeruginosa 1397, Escherichia coli ATCC 25922), Gram-positive (Enterococcus faecalis ATCC 29212, Bacillus subtilis IC 12488) bacterial as well as fungal (Candida krusei 963) strains. The obtained results suggested that the antimicrobial activity of IO-NPs is dependent on the metallic ions concentrations and on the microbial growth state, either planktonic or adherent. The obtained IO-NPs exhibited no cytotoxic effect on HeLa cells at the active antimicrobial concentrations.
\end{abstract}

\section{Introduction}

During the last decade, there has been an increasing interest in developing and understanding of matter at nanometric scale $[1,2]$. The progress made in the area of nanotechnology in the last years has allowed the scientists to develop and characterize materials with outstanding properties and nanometric sizes [3]. The field of nanomaterials has been the centre of attention in the scientific community due to the unique and remarkable properties exhibited by materials when studied at nanometric scale. Recent studies state that inorganic materials are the core of future developments in industrial and technological applications [4]. During the past few years, the most studied materials were the ones with magnetic behaviour due to their promising applications in areas such as magnetic data storage [5], gas sensors [6$8]$, tunnelling magneto-resistance (TMR) $[9,10]$, ferrofluid, targeted drug delivery, contrast agents in magnetic resonance imaging [11], hyperthermia, magnetic field assisted radionuclide therapy [12] as well as magnetically separable absorbents [13]. Amongst materials with magnetic properties, iron oxide nanoparticles are most suitable for biomedical applications due to their proven biocompatibility. Magnetite and maghemite, the most common iron oxide nanoparticles, have been the subject of intensive studies because at nanometric scale they exhibit unique physical and chemical particularities [14].

Due to the increased resistance to existing antibiotics of various microorganisms, many researchers have turned towards engineered nanoparticles for finding a solution. 
The ability of pathogenic bacteria and fungi to resist antimicrobial agents, by genetic mechanisms and by phenotypic resistance due to the biofilm growing state, has emerged in the recent years and became a major health problem. Recent studies reported various metal nanoparticles to exhibit antimicrobial effects [15]. Iron oxide nanoparticles, due to their small size, superparamagnetic properties and controllable parameters, can interact directly with various microorganisms without posing a risk for human body. These nanoparticles can interact directly with the microbial cells causing damage to the envelope and thus rendering the bacterial cell defenceless or, in some cases, disrupting the metabolic functions and causing the destruction of the cell [16].

Magnetic iron oxide nanoparticle are increasingly being developed for use in research and biomedical applications, such as targeted transport of antimicrobial drug. The fundamental understanding on the influence of magnetic iron oxide nanoparticle on cellular growth and functions is very important for further biological applications. The comparative study on growth of different bacteria the influence of magnetic iron oxide were revealed the effect of magnetic iron oxide nanoparticle on bacterial growth.

This study focuses on the investigation of the antimicrobial behavior of iron oxide nanoparticles (IO-NPs) prepared by an adapted co-precipitation method [17-21] and on the analysis of $\mathrm{M}-\mathrm{H}$ hysteresis loops at room temperature. Transmission electron microscopy studies have been conducted to obtain information about size, structure and morphology of the fabricated IO-NPs. The antimicrobial activity of the IONPs was investigated by qualitative and quantitative assays and their cytotoxic effect was assessed on HeLa cells. In this study, the IO-NPs were tested in aqueous suspension against a large spectrum of microbial strains, both by antimicrobial susceptibility standard methods (agar diffusion and minimal inhibitory concentration assay) as well as by methods aimed to investigate the efficiency of IO-NPs against biofilm embedded cells, which are much more resistant to different stress factors, including antimicrobials, than their planktonic counterparts.

\section{Experimental Section}

2.1. Materials. Ferrous chloride tetrahydrate $\left(\mathrm{FeCl}_{2} \cdot 4 \mathrm{H}_{2} \mathrm{O}\right)$, ferric chloride hexahydrate $\left(\mathrm{FeCl}_{3} \cdot 6 \mathrm{H}_{2} \mathrm{O}\right)$, natrium hydroxide $(\mathrm{NaOH})$, and hydrochoric acid $(\mathrm{HCl})$ were purchased from Merck. Deionized water was used in the synthesis of nanoparticles and for rinsing of clusters.

2.2. Synthesis of Iron Oxide Ferrofluid and Characterization. The synthesis of iron oxide ferrofluid was carried out as reported in other papers [22]. Transmission electron microscopy (TEM) images for these samples were recorded using a FEI Tecnai 12 equipped with a low dose digital camera from Gatan. The specimen for TEM imaging was prepared by ultramicrotome to get thin section of about $60 \mathrm{~nm}$. The powder was embedded in an epoxy resin (polaron 612) before microtomy. The magnetic properties of the samples were measured using a superconducting quantum interference device (MPMS magnetometer) at room temperature.

2.3. Cytotoxicity Assay. Quantification of cell viability was performed using propidium iodide (PI) and fluorescein diacetate $(\mathrm{FdA})$. Briefly, $5 \times 10^{4} \mathrm{HeLa}$ cells were seeded in each well of a plate with 24 wells and after $24 \mathrm{~h}$ were treated with $10 \mu \mathrm{L}$ of $\mathrm{Fe}^{2+} / \mathrm{Fe}^{3+}$ of 0.03 final concentration. The effects on cell viability were evaluated after $24 \mathrm{~h}$ by adding $100 \mu \mathrm{L}$ PI $(0.1 \mathrm{mg} / \mathrm{mL})$ and $100 \mu \mathrm{L} \mathrm{FdA}(0.1 \mathrm{mg} / \mathrm{mL})$ and fluorescence was quantified using Observer D1 Carl Zeiss microscope. All cells from several fields were counted and cell viability was established by the ratio between viable (green) and dead cells (red) [23].

\subsection{The In Vitro Antibacterial and Antibiofilm Activity}

Assessment of the Antimicrobial and Antipathogenic Activity of the New Oxides. The in vitro qualitative screening of the antimicrobial activity was carried out by an adapted agar diffusion technique using a bacterial suspension of 0.5 McFarland density obtained from 24 hour cultures [24, 25]. The antimicrobial activity of the IO-NPs was tested against Gram-negative (Pseudomonas aeruginosa 1397, Escherichia coli ATCC 25922), Gram-positive (Enterococcus faecalis ATCC 29212, Bacillus subtilis (IC 12488) bacterial as well as fungal (Candida krusei 963) strains. The microbial strains identification was confirmed by aid of VITEK II automatic system. VITEK cards for identification and susceptibility testing (GNS-522) were inoculated and incubated according to the manufacturer's recommendations. IO-NPs were tested starting with a concentration of $10 \mathrm{mg} / \mathrm{mL}$.

Qualitative Screening of the IO-NPs Antimicrobial Activity. A volume of $10 \mu \mathrm{L}$ of each tested IO-NPs sample of $10 \mathrm{mg} / \mathrm{mL}$ concentration was spotted on Muller Hinton agar for bacteria and Yeast Peptone Glucose (YPG) agar for fungi, previously seeded with the microbial inocula of $0.5 \mathrm{MacF}$ arland density. The inoculated plates were incubated for $24 \mathrm{~h}$ at $37^{\circ} \mathrm{C}$. The antimicrobial activity was assessed by measuring the growth inhibition zones diameters expressed in $\mathrm{mm}$ [26]. Following the results of the qualitative screening, only the microbial strains proving to be susceptible have been further tested in the quantitative assay.

The quantitative assay of the minimal inhibitory concentration $(M I C, \mu \mathrm{g} / \mathrm{mL}$ ) was based on liquid medium two-fold microdilutions and performed in 96 multi-well plates. For this purpose, serial binary dilutions of the tested compounds (ranging between 5 and $0.01 \mu \mathrm{g} / \mathrm{mL}$ ) were performed in a $200 \mu \mathrm{L}$ volume of nutrient broth (for bacteria)/YPG (for fungi) and each well was seeded with $20 \mu \mathrm{L}$ microbial inocula of $0.5 \mathrm{McF}$ arland density. The plates were incubated for $24 \mathrm{~h}$ at $37^{\circ} \mathrm{C}$, the antimicrobial effect being assessed by measuring the absorbance of the obtained cultures at $620 \mathrm{~nm}$ [26]. The lower absorbance value was, the more intensive the antimicrobial effect is.

The antibiofilm activity of the IO-NPs was tested by the microtiter method. For this purpose, the microbial strains 


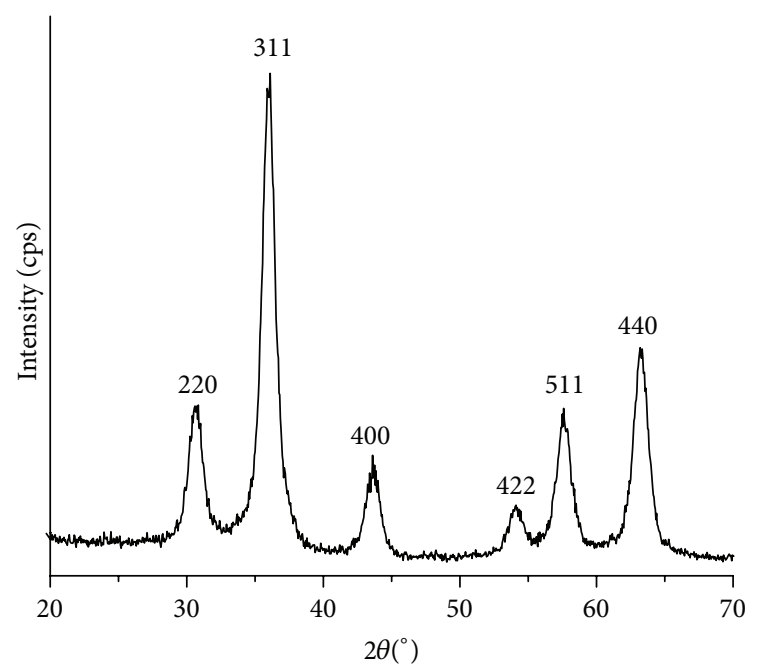

FIGURE 1: XRD patterns of maghemite $\gamma-\mathrm{Fe}_{2} \mathrm{O}_{3}$ nanoparticles obtained by coprecipitation method.

have been grown in the presence of two-fold serial dilutions of the tested compounds (ranging between 5 and $0.01 \mu \mathrm{g} / \mathrm{mL}$ ), performed in liquid nutrient broth/YPG, distributed in 96well plates and incubated for 24 hours at $37^{\circ} \mathrm{C}$. At the end of the incubation period, the plastic wells were emptied, washed three times with phosphate buffered saline (PBS), fixed with cold methanol and stained with $1 \%$ violet crystal solution for 30 minutes. The biofilm formed on plastic wells was resuspended in 30\% acetic acid. The intensity of the colored suspensions was assessed by measuring the absorbance at $492 \mathrm{~nm}$ [26-28]. The lower absorbance value was, the more intensive the antibiofilm effect was considered.

\section{Results and Discussion}

Figure 1 shows the XRD patterns of the iron oxide particles. The $2 \theta$ values of the peaks are compared with the standard data for $\gamma-\mathrm{Fe}_{2} \mathrm{O}_{3}$. Diffraction peaks at (220), (311), (400), (422), (511) and (440) are readily recognized from the XRD pattern in the sample. The XRD peaks of iron oxide nanoparticles (Figure 1) can be indexed into the spinel cubic lattice type with a lattice of $0.835 \mathrm{~nm}$. This value of lattice parameter is in agreement with the values of the standard data (JCPDS no. 4-755). The refinement of XRD spectra indicated that no other phases except the maghemite are detectable. The XRD showed a finite broadening of the diffraction lines. The particle size calculated using the Scherer [29] formula was estimated at around $7.7 \mathrm{~nm}$.

Figure 2(a) shows TEM picture of iron oxide nanoparticles (IO-NPs), clearly showing that the product is entirely composed of crystals with a relatively uniform, spherical morphology. Grain size distribution was determined by measuring the mean diameter, $D$, of about 500 particles on the micrographs (Figure 2(c)). TEM images indicate a very uniform size distribution of iron oxide nanoparticles. The average grain size of the monodisperse nanoparticles is $7 \pm 0.2 \mathrm{~nm}$. Figure 2(b) also shows the selected area electron diffraction (SAED) pattern recorded from an area containing a large number of nanoparticles and high-resolution TEM picture. The rings in the SAED pattern can be indexed as the (220), (311), (400), (422), (511) and (440) reflections of the cubic maghemite in agreement with the XRD results.

The magnetic properties of the prepared IO-NPs were monitored by measuring the hysteresis loops at a temperature of $300 \mathrm{~K}$. Magnetization curves (M-H loop) for the magnetic IO-NPs measured at room temperature were presented in Figure 3. At the maximum applied magnetic field of $10 \mathrm{kOe}$ the magnetization did not reach saturation no hysteresis loops can be observed (no remanent magnetization and zero coercivity fields) $[30,31]$. This behavior indicated that the IO-NPs were superparamagnetic at room temperature with a calculated saturation magnetization of $18.1 \mathrm{emu} / \mathrm{g}$. The iron oxide nanoparticles sample shows a saturation magnetization at lower than the reported values for the saturation magnetization of bulk maghemite $\left(\gamma-\mathrm{Fe}_{2} \mathrm{O}_{3}\right)$ [32]. This trend could be attributed to the particles sizes effect. The magnetic nanoparticles are so small that they may be considered to have a single magnetic domain [33]. In his studies, Hergt et al., showed that the saturation magnetizations, Ms, decreases with decreasing particle size [34]. Analysis the hysteresis loops of the IO-NPs, at $300 \mathrm{~K}$ confirms average size and particle size distribution of IO-NPs already obtained from TEM and XRD analyses. Combination of magnetic properties and antimicrobial features of iron oxide nanoparticles, designates these nanoparticles for exploitation in biomedical applications, such as targeted transport of antimicrobial drug. With regard to their unique magnetic and antibacterial properties, the iron oxide nanoparticles can be exploited at lower concentrations so that their toxic effect on human cells to be minimized.

A great advantage of the tested compound is that they are not cytotoxic at the active concentrations, as reveal by the cytotoxicity assay, demonstrating no cytotoxic effect of the IO-NPs (Figure 4).

The microbial species of clinical interest often involved in biofilm associated diseases are belonging to a very large spectrum, from the Gram positive (S. epidermidis and $S$. aureus), and Gram negative ( $P$. aeruginosa, E. coli) bacterial strains to different members of the Candida genus [35]. The understanding of microbial infections related to the biofilm development on tissues or indwelling devices was possible by using different qualitative and quantitative in vitro specific assays. In our study we have also used both assays for studying the susceptibility of microbial cells grown in suspension, known as planktonic cells, as well as of those developed in biofilms, called adherent or sessile cells.

The antimicrobial activity of the IO-NPs has been studied on strains belonging to common bacterial pathogens, that is, the Gram-negative, P. aeruginosa, E. coli, Gram-positive E. faecalis, B. subtilis, and a yeast strain of C. krusei.

After the qualitative screening, the microbial strains which proved to be susceptible to the tested nanoparticles have been investigated in the quantitative assay for establishing the MIC value. The highest tested concentration was of $5 \mathrm{mg} / \mathrm{mL}$, because at higher concentrations, the colored iron oxide suspension would have interfered with the reading 


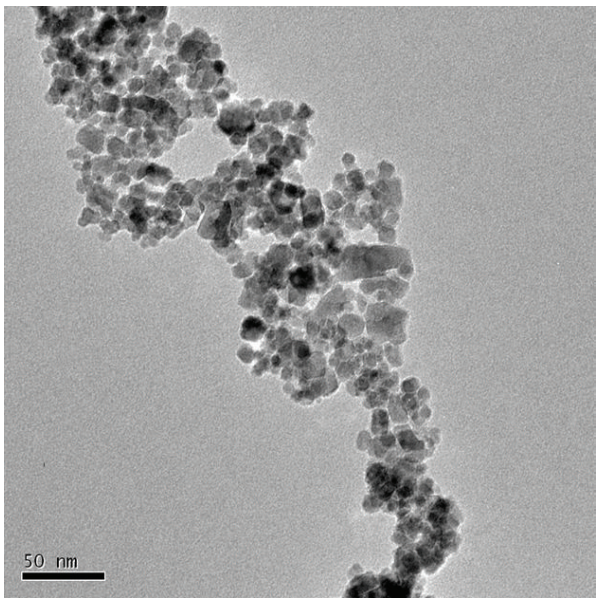

(a)

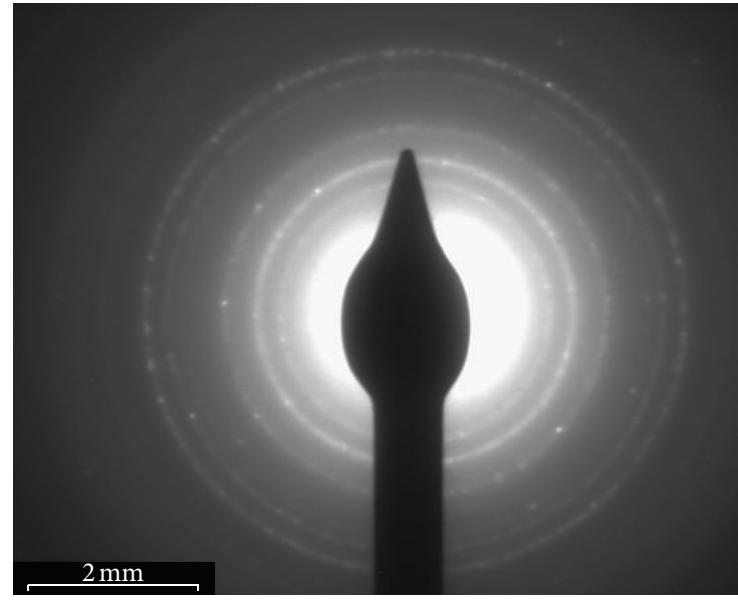

(b)

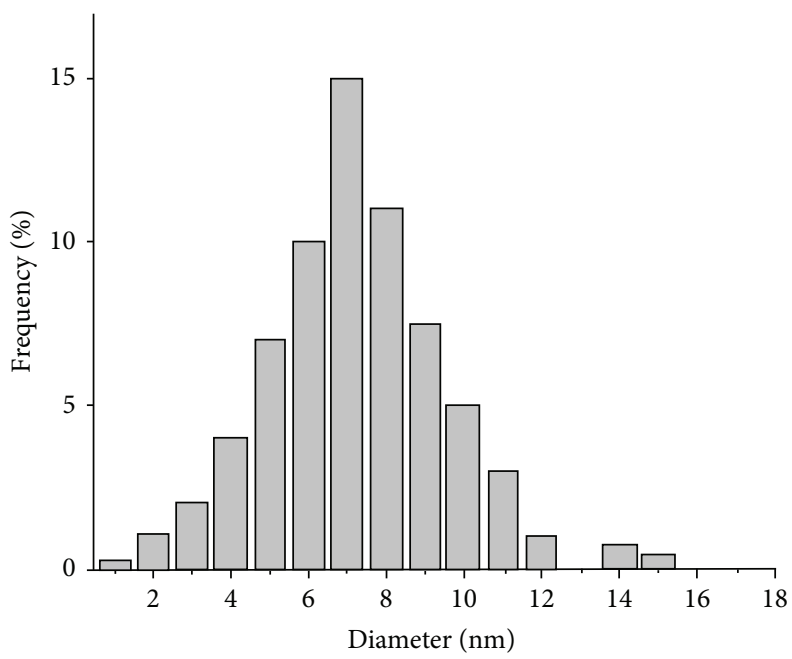

(c)

FIGURE 2: Bright field TEM picture showing a homogeneous distribution of iron oxide nanoparticles (a), SAED pattern from a region including a large number of nanoparticles (b), and size distribution of IO-NPs (c).

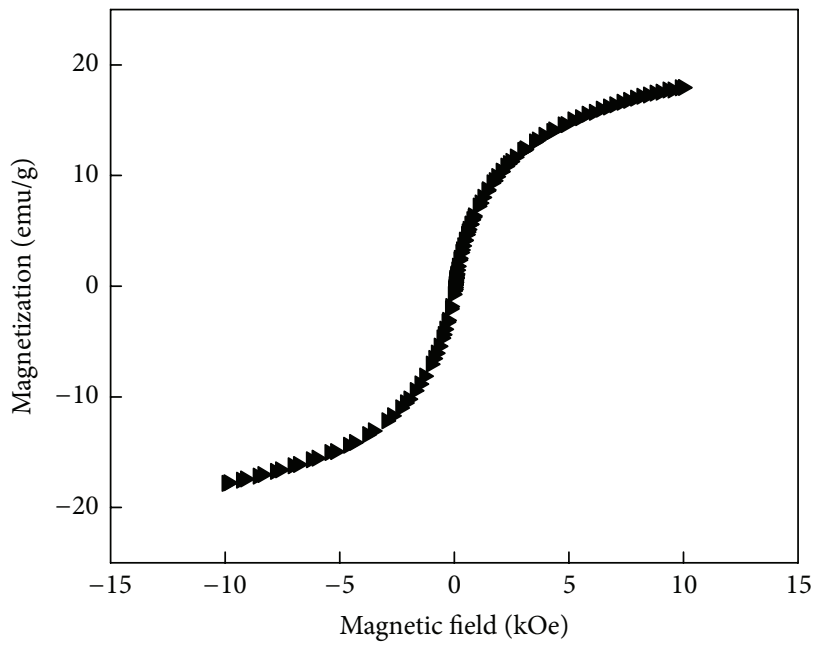

(a)

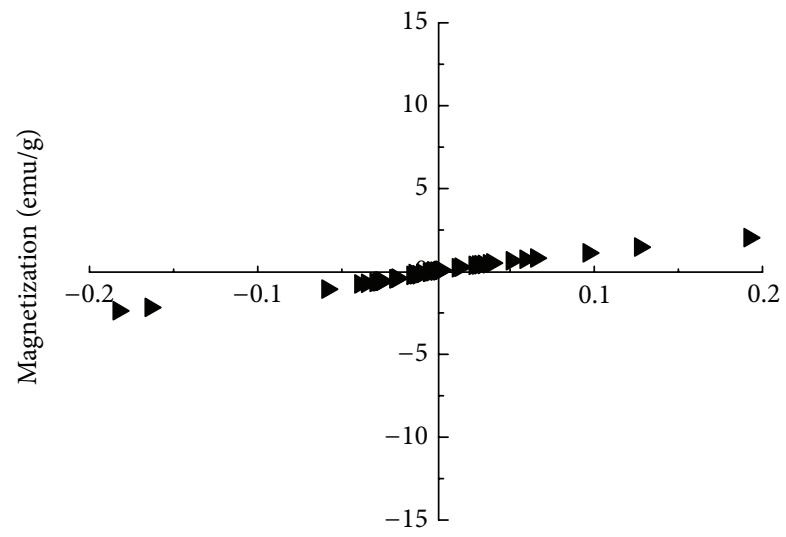

Magnetic field (kOe)

(b)

Figure 3: M-H curves of iron oxide nanoparticles at room temperature (a). The low field parts (b). 


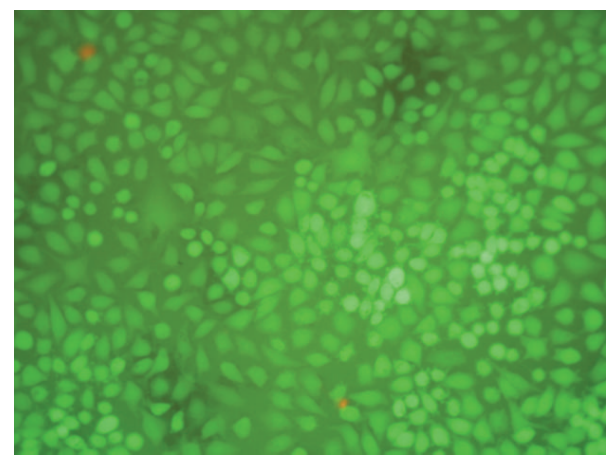

FIGURE 4: Fluorescence microscopy image revealing the absence of the cytotoxic effect on HeLa cells (no dead cell stained in red) after $24 \mathrm{~h}$ treatment with $200 \mu \mathrm{L}$ of iron oxide $(\times 100)$.

IO-NPs

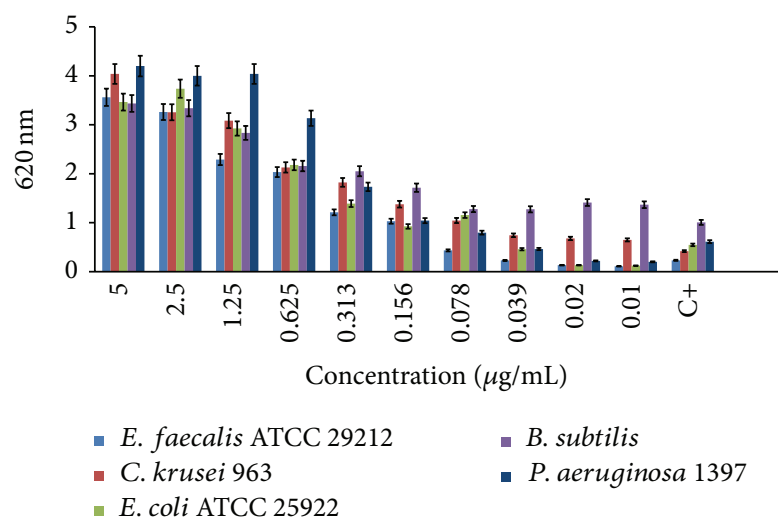

FIgURE 5: The quantitative assay of the inhibitory effect of the IONPs on different microbial strains, quantified by the absorbance measurements at $620 \mathrm{~nm} . \mathrm{C}=$ positive control, represented by the microbial culture grown in the absence of IO-NPs.

of the absorbance values at $620 \mathrm{~nm}$. The quantitative assay showed that the IO-NPs generally stimulated the growth of all microbial cells in suspension from the highest tested concentrations of $5 \mathrm{mg} / \mathrm{mL}$ to $0.039 \mathrm{mg} / \mathrm{mL}$, as demonstrated by the absorbance measurements at $620 \mathrm{~nm}$ of the obtained cultures (Figure 5).

A bactericidal effect has been observed at low IO-NPs concentrations, that is, from 0.039 to $0.01 \mathrm{mg} / \mathrm{mL}$ against $E$. faecalis ATCC 29212 and P. aeruginosa 1397, from 0.02 to 0.01 against $E$. coli ATCC 25922. The IO-NPs, irrespective to the tested concentration exhibited no inhibitory effect on $C$. krusei 963 and B. subtilis IC 12488 growth.

Metal oxide nanoparticles, including magnetite, have been proved to have inherent antimicrobial properties, which are enhanced, or occur, when the materials are in the nanometer size and in relation to surface area [36]. Since nanoparticles can be smaller in size than bacterial pores, they have a unique ability of crossing the cell membrane, disrupting its function or interfering with nucleic acid or protein synthesis [37]. Our study demonstrated that IO-NPs are rather exhibiting a stimulatory effect on the microbial growth especially at high concentrations. The stimulatory effect of iron oxide nanoparticles on the microbial growth was also reported by other authors, that is, on E. coli [38] or E. coli, $P$. aeruginosa and E. faecalis, C. albicans [39]. These results could be explained by the ability of the microbial strains to use the iron oxide as a metabolic source of iron, which is known to positively regulate the microbial growth rate and other physiological processes [40].

However, at lower concentrations ranging from 39 to $10 \mu \mathrm{g} / \mathrm{mL}$, they inhibited the growth of E. faecalis, $P$. aeruginosa and E. coli strains. Our results come into agreement with other studies showing that iron oxide nanoparticles substantially inhibited the growth of E. coli and S. aureus at concentrations comparable with those used in our study, from 10 to $100 \mu \mathrm{g} / \mathrm{mL}$ [41]. $\mathrm{Fe}_{2} \mathrm{O}_{3}$ nanoparticles have been shown to exhibit the least bactericidal activity, when compared with other metal oxides, like $\mathrm{ZnO}$ or $\mathrm{CuO}$ [42]. The antibacterial activity of composite nanoparticles containing iron oxide, zinc oxide and zinc ferrite phases has been shown to be proportional with the weight ratio $[\mathrm{Zn}] /[\mathrm{Fe}]$ of the composite nanoparticle, on Staphylococcus aureus and Escherichia coli, so the lowest the iron concentration, the most intensive the antimicrobial activity [37].

Studies in the last decades on microbial adherence to different substrata led to the conclusion that the survival of microorganisms in the natural habitats, including medical ecosystems, is dependent on their capacity to adhere to different surfaces/substrata and form biofilms. A biofilm is a sessile microbial community composed of cells embedded in a matrix of extracellular polymeric substances attached to a substratum or interface. The matrix is primarily of microbial origin and the cells encased in this matrix present a modified phenotype, being metabolically more efficient and well protected, exhibiting resistance to different stress factors, including host defense mechanisms and antibiotics [43].

The quantitative assay of the inhibitory effect of IO-NPs on biofilms developed on the inert substratum by different microbial strains, quantified by the absorbance measurements at $490 \mathrm{~nm}$ are presented in Figure 6. The highest tested concentration (i.e., $5 \mathrm{mg} / \mathrm{mL}$ ) exhibited a strong stimulatory effect on the biofilm development by all tested microbial strains, this effect drastically decreasing in case of the other tested concentrations (Figure 6). The tested IO-NPs exhibited only a slight antibiofilm activity for the same strains susceptible in the planktonic state, that is, E. faecalis $29212(0.625$ to $0.01 \mathrm{mg} / \mathrm{mL})$, P. aeruginosa 1397 (2.5 to $0.01 \mathrm{mg} / \mathrm{mL}$ ), and E. coli ATCC 25922 (0.156 to $0.01 \mathrm{mg} / \mathrm{mL}$ ) strains (Figure 6). Comparing the range of active concentrations of IO-NPs against planktonic and respectively adherent strains, it could be observed that the antibiofilm activity of the obtained nanoparticles is maintained on a wider range of concentrations.

\section{Conclusions}

Iron oxide nanoparticles were synthesized by a facile and rapid coprecipitation method. The prepared IO-NPs were characterized by various techniques. The TEM images show 


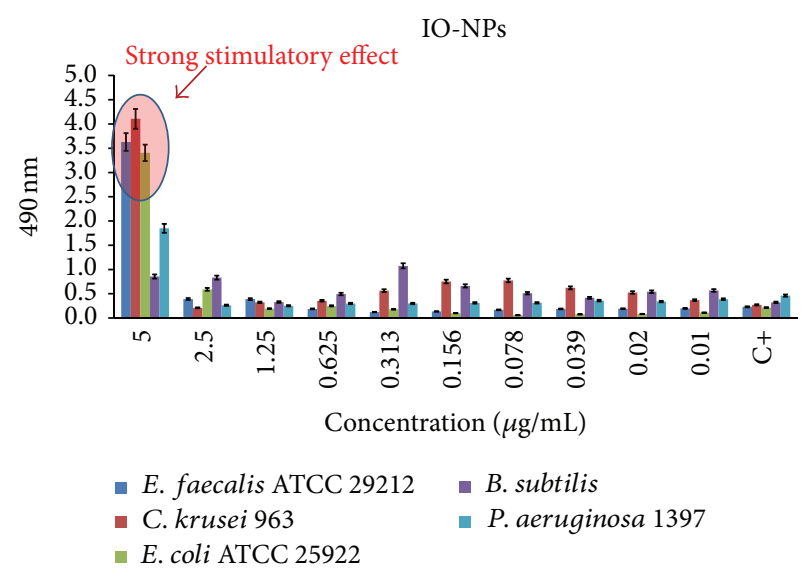

FIGURE 6: The quantitative assay of the inhibitory effect of IO-NPs on biofilms developed on the inert substratum by different microbial strains, quantified by the absorbance measurements at $490 \mathrm{~nm}$.

well-crystallized magnetic IO-NPs with average size of $7 \pm 0.2 \mathrm{~nm}$ and narrow size distributions without particle agglomeration. The hysteresis loop of IO-NPs demonstrates a superparamagnetic behavior. The IO-NPs exhibited a strong stimulatory effect directly correlated with high concentrations of the tested nanoparticles, both on the growth of microbial strains in suspension and in adherent state, probably due to the potential of these strains to use the iron oxide as a source of iron ions required for the microbial metabolism and pathogenicity. However, the tested iron oxide nanoparticles inhibited on a certain range of concentrations the ability of $E$. faecalis, $P$. aeruginosa, and E. coli strains to grow, both in suspension and in biofilm state, results demonstrating their potential use in the design of antibacterial and antibiofilm materials and surfaces, for biomedical applications, exhibiting also the great advantage of reduced cytotoxicity on eukaryotic host cells and tissues. The combination of magnetic properties and antimicrobial features designates the iron oxide nanoparticles to be possibly used in applications such as clinical infections and local antimicrobial therapy. On the other hand, these nanoparticles could be used for targeted transport of an antimicrobial agent and its subsequent removal by means of an external magnetic field.

\section{Acknowledgments}

The financial and encouragement support provided by the Ministry of Educations of the Romania, Project no. C2-06 under Program CEA-IFA, POSDRU/107/1.5/S/76813 (DocInvest), by the 94/PN2/PCCA (AntiBioTube) program and by the scholarship of the French government via the cultural section of the French Embassy.

\section{References}

[1] G. Nicoara, D. Fratiloiu, M. Nogues, J. L. Dormann, and F. Vasiliu, "Ni-Zn ferrite nanoparticles prepared by ball milling," Materials Science Forum, vol. 235-238, no. 1, pp. 145-150, 1997.
[2] D. Prodan, C. Chanéac, E. Tronc et al., "Adsorption phenomena and magnetic properties of $\gamma-\mathrm{Fe}_{2} \mathrm{O}_{3}$ nanoparticles," Journal of Magnetism and Magnetic Materials, vol. 203, no. 1-3, pp. 63-65, 1999.

[3] D. Prodan, V. V. Grecu, M. N. Grecu, E. Tronc, and J. P. Jolivet, "Electron spin resonance in $\gamma$ - $\mathrm{Fe}_{2} \mathrm{O}_{3}$ nanoparticles dispersed in a polymer matrix," Measurement Science and Technology, vol. 10, no. 9, pp. L41-L43, 1999.

[4] A. Figuerola, R. Di Corato, L. Manna, and T. Pellegrino, "From iron oxide nanoparticles towards advanced iron-based inorganic materials designed for biomedical applications," Pharmacological Research, vol. 62, no. 2, pp. 126-143, 2010.

[5] M. L. Yan, X. Z. Li, L. Gao et al., "Fabrication of nonepitaxially grown double-layered $\mathrm{FePt}$ :C/FeCoNi thin films for perpendicular recording," Applied Physics Letters, vol. 83, no. 16, pp. 3332$3334,2003$.

[6] D. Predoi, C. S. Ciobanu, M. Radu et al., "Hybrid dextran-iron oxide thin films deposited by laser techniques for biomedical applications," Materials Science and Engineering C, vol. 32, no. 2, pp. 296-302, 2012.

[7] O. K. Tan, W. Zhu, Q. Yan, and L. B. Kong, "Size effect and gas sensing characteristics of nanocrystalline ${ }_{x} \mathrm{SnO}_{2}-(1-x) \alpha-\mathrm{Fe}_{2} \mathrm{O}_{3}$ ethanol sensors," Sensors and Actuators B, vol. 65, no. 1, pp. 361365, 2000.

[8] E. Andronescu, M. Costache, C. S. Ciobanu, A. M. Prodan, and D. Predoi, "Biocompatibility studies of iron-oxide-dextrin thin films," Revista de Chimie, vol. 61, no. 10, pp. 925-928, 2010.

[9] Y. Peng, C. Park, and D. E. Laughiin, " $\mathrm{Fe}_{3} \mathrm{O}_{4}$ thin films sputter deposited from iron oxide targets," Journal of Applied Physics, vol. 93, no. 10, pp. 7957-7959, 2003.

[10] C. S. Ciobanu, E. Andronescu, L. Pall, S. L. Iconaru, E. Gyorgy, and D. Predoi, "Physico-chemical properties of iron-oxidedextnn thin films," Revista de Chimie, vol. 61, no. 12, pp. 12071211, 2010.

[11] M. Bystrzejewski, A. Huczko, and H. Lange, "Arc plasma route to carbon-encapsulated magnetic nanoparticles for biomedical applications," Sensors and Actuators B, vol. 109, no. 1, pp. 81-85, 2005.

[12] Z. Sun, L. Wang, P. Liu et al., "Magnetically motive porous sphere composite and its excellent properties for the removal of pollutants in water by adsorption and desorption cycles," Advanced Materials, vol. 18, no. 15, pp. 1968-1971, 2006.

[13] A. R. Jalilian, A. Panahifar, M. Mahmoudi, M. Akhlaghi, and A. Simchi, "Preparation and biological evaluation of $\left[{ }^{67} \mathrm{Ga}\right]$-labeled-superparamagnetic nanoparticles in normal rats," Radiochimica Acta, vol. 97, no. 1, pp. 51-56, 2009.

[14] Y. Xiong, J. Ye, X. Gu, and Q. Chen, "Synthesis and magnetic properties of iron oxide nanoparticles/C and $\alpha$-Fe/iron oxide nanoparticles/C composites," Journal of Magnetism and Magnetic Materials, vol. 320, no. 3-4, pp. 107-112, 2008.

[15] M. P. Moralesa, O. Bomati-Miguela, R. Perez de Alejob, J. Ruiz-Cabellob, S. Veintemillas-Verdaguera, and K. O'Gradyc, "Contrast agents for MRI based on iron oxide nanoparticles prepared by laser pyrolysis," Journal of Magnetism and Magnetic Materials, vol. 266, pp. 102-109, 2003.

[16] F. Furno, K. S. Morley, B. Wong et al., "Silver nanoparticles and polymeric medical devices: a new approach to prevention of infection?" Journal of Antimicrobial Chemotherapy, vol. 54, no. 6, pp. 1019-1024, 2004.

[17] R. Massart, "Magnetic fluids and process for obtaining them," US Patent 4329241, 1982. 
[18] R. Massart, "Preparation of aqueous magnetic liquids in alkaline and acidic media," IEEE Transactions on Magnetics, vol. 17, no. 2, pp. 1247-1248, 1981.

[19] R. Massart, J. Roger, and V. Cabuil, "New trends in chemistry of magnetic colloids: polar and non polar magnetic fluids, emulsions, capsules and vesicles," Brazilian Journal of Physics, vol. 25, no. 2, pp. 135-141, 1995.

[20] D. Predoi and C. M. Valsangiacom, "Thermal studies of magnetic spinel iron oxide in solution," Journal of Optoelectronics and Advanced Materials, vol. 9, no. 6, pp. 1797-1799, 2007.

[21] D. Zins, V. Cabuil, and R. Massart, "New aqueous magnetic fluids," Journal of Molecular Liquids, vol. 83, no. 1-3, pp. 217-232, 1999.

[22] C. S. Ciobanu, S. L. Iconaru, E. Gyorgy et al., "Biomedical properties and preparation of iron oxide-dextran nanostructures by MAPLE technique," Chemistry Central Journal, vol. 6, p. 17, 2012.

[23] A. M. Grumezescu, E. Andronescu, A. Ficai, C. Bleotu, and M. C. Chifiriuc, "Chitin based biomaterial for antimicrobial therapy: fabrication, characterzation and in vitro profile based intercation with eukaryotic and prokaeryotic cells," Biointerface Research in Applied Chemistry, vol. 2, p. 446, 2012.

[24] A. M. Grumezescu, E. Andronescu, A. Ficai et al., "Magnetic nanofluid with antitumoral properties," Letters in Applied NanoBioScience, vol. 1, p. 56, 2012.

[25] A. M. Grumezescu, A. Holban, E. Andronescu, A. Ficai, C. Bleotu, and M. C. Chifiriuc, "Microbiological applications of a new water dispersible magnetic nanobiocomposite," Letters in Applied NanoBioScience, vol. 4, p. 83, 2012.

[26] C. Saviuc, A. M. Grumezescu, C. M. Chifiriuc et al., "Hybrid nanosystem for stabilizing essential oils in biomedical applications," Digest Journal of Nanomaterials and Biostructures, vol. 6, no. 4, pp. 1657-1666, 2011.

[27] C. Saviuc, A. M. Grumezescu, A. Holban et al., "Phenotypical studies of raw and nanosystem embedded Eugenia carryophyllata buds essential oil antibacterial activity on Pseudomonas aeruginosa and Staphylococcus aureus strains," Biointerface Research in Applied Chemistry, vol. 1, no. 3, pp. 111-118, 2011.

[28] C. Saviuc, I. Marinas, A. M. Grumezescu et al., "Phytochemical composition of the fennel fruits essential oil and its influnence on prokariotic cells growth and pathogenic features," Biointerface Research in Applied Chemistry, vol. 2, p. 300, 2012.

[29] R. M. Cornell and U. Schertmann, Iron Oxides in the Laboratory: Preparation and Characterisation, VCH, Weinheim, Germany, 1991.

[30] K. V. P. M. Shafi, A. Gedanken, R. Prozorov, and J. Balogh, "Sonochemical preparation and size-dependent properties of nanostructured $\mathrm{CoFe}_{2} \mathrm{O}_{4}$ particles," Chemistry of Materials, vol. 10, no. 11, pp. 3445-3450, 1998.

[31] R. Vijayakumar, Y. Koltypin, I. Felner, and A. Gedanken, "Sonochemical synthesis and characterization of pure nanometersized $\mathrm{Fe}_{3} \mathrm{O}_{4}$ particles," Materials Science and Engineering A, vol. 286, no. 1, pp. 101-105, 2000.

[32] E. du Tremolet deLacheisserie, D. Gignoux, and M. Schlenker, Magnetism: Materialsandapplications, Springer, New York, NY, USA, 2005.

[33] D. H. Han, J. P. Wang, and H. L. Luo, "Crystallite size effect on saturation magnetization of fine ferrimagnetic particles," Journal of Magnetism and Magnetic Materials, vol. 136, no. 1-2, pp. 176-182, 1994.

[34] R. Hergt, W. Andrae, C. G. d'Ambly et al., "Physical limits of hyperthermia using magnetite fine particles," IEEE Transactions on Magnetics, vol. 34, no. 5, pp. 3745-3754, 1998.
[35] V. Lazăr and M. C. Chifiriuc, "Medical significance and new therapeutical strategies for biofilm associated infections," Roumanian Archives of Microbiology \& Immunology, vol. 69, no. 3, pp. 125-138, 2010.

[36] E. Taylor and T. J. Webster, "Reducing infections through nanotechnology and nanoparticles," International Journal of Nanomedicine, vol. 6, pp. 1463-1473, 2011.

[37] T. Gordon, B. Perlstein, O. Houbara, I. Felner, E. Banin, and S. Margel, "Synthesis and characterization of zinc/iron oxide composite nanoparticles and their antibacterial properties," Colloids and Surfaces A, vol. 374, no. 1-3, pp. 1-8, 2011.

[38] S. Nath, C. Kaittanis, V. Ramachandran, N. S. Dalal, and J. M. Perez, "Synthesis, magnetic characterization, and sensing applications of novel dextran-coated iron oxide nanorods," Chemistry of Materials, vol. 21, no. 8, pp. 1761-1767, 2009.

[39] M. Singh, S. Singh, S. Prasad, and I. S. Gambhir, "Nanotechnology in medicine and antibacterial effect of silver nanoparticles," Digest Journal of Nanomaterials and Biostructures, vol. 3, pp. 115-122, 2008.

[40] A. Azam, A. S. Ahmed, M. Oves, M. S. Khan, S. S. Habib, and A. Memic, "Antimicrobial activity of metal oxide nanoparticles against Gram-positive and Gram-negative bacteria: a comparative study," International Journal of Nanomedicine, vol. 7, pp. 6003-6009, 2012.

[41] S. A. Mahdy, Q. J. Raheed, and P. T. Kalaichelvan, "Antimicrobial activity of zero-valent iron nanoparticles," International Journal of Modern Engineering Research, vol. 2, no. 1, pp. 578-581, 2012.

[42] A. K. Horst, "Antimicrobial effects of metal oxide nanoparticles," The NNIN REU Research Accomplishmnents Biological Applicantions, 2009.

[43] C. Chifiriuc, V. Lazăr, C. Bleotu et al., "Bacterial adherence to the cellular and inert substrate in the presence of $\mathrm{CoFe}_{2} \mathrm{O}_{4}$ and $\mathrm{Fe}_{3} \mathrm{O}_{4}$ /oleic acid-core/shell," Digest Journal of Nanomaterials and Biostructures, vol. 6, no. 1, pp. 37-42, 2011. 

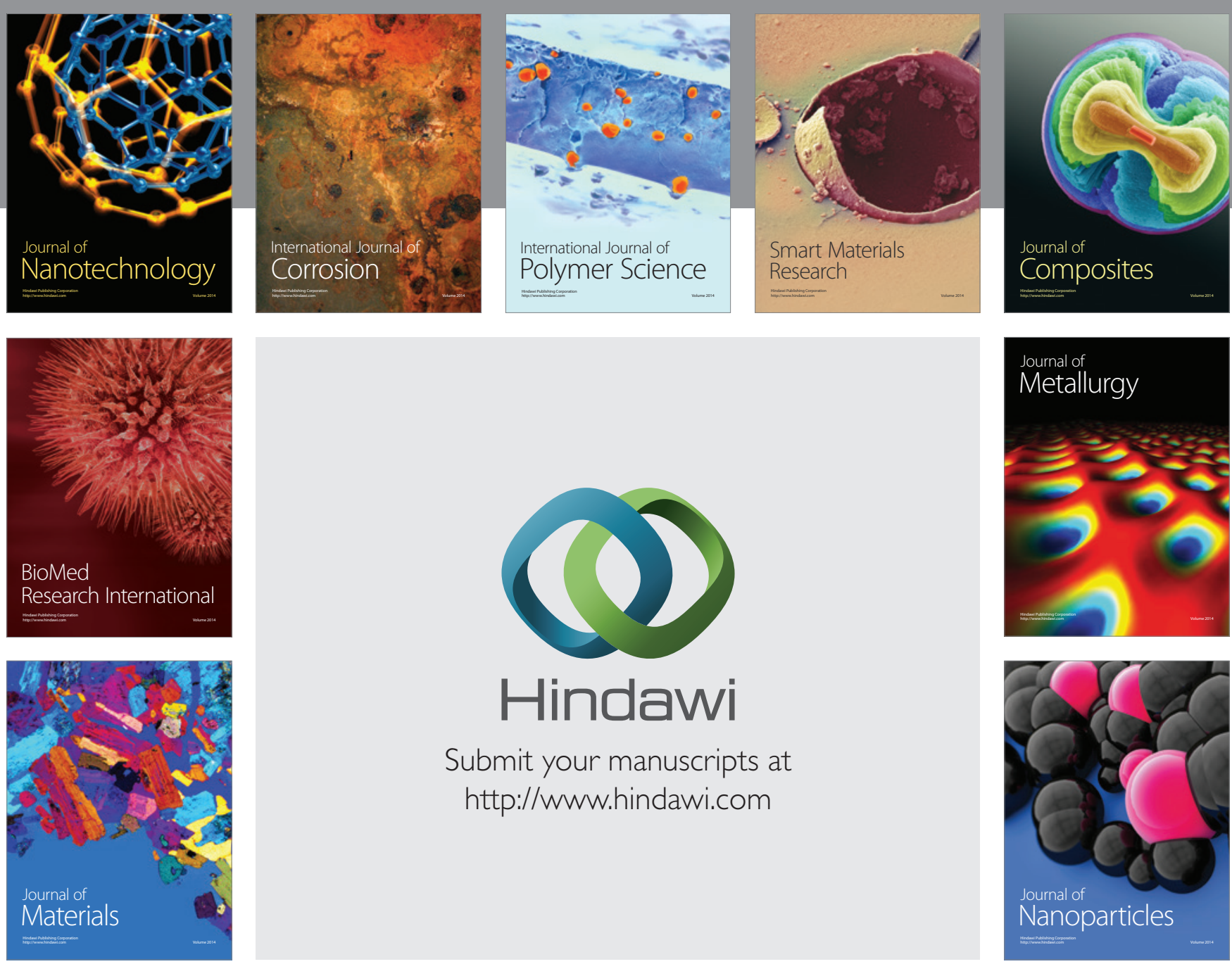

Submit your manuscripts at http://www.hindawi.com
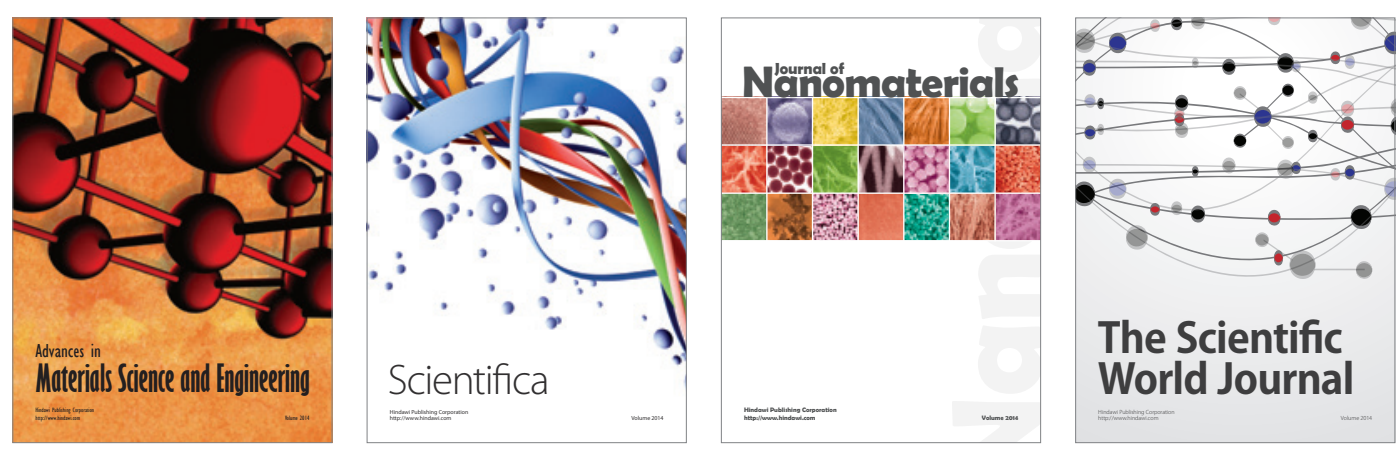

\section{The Scientific World Journal}
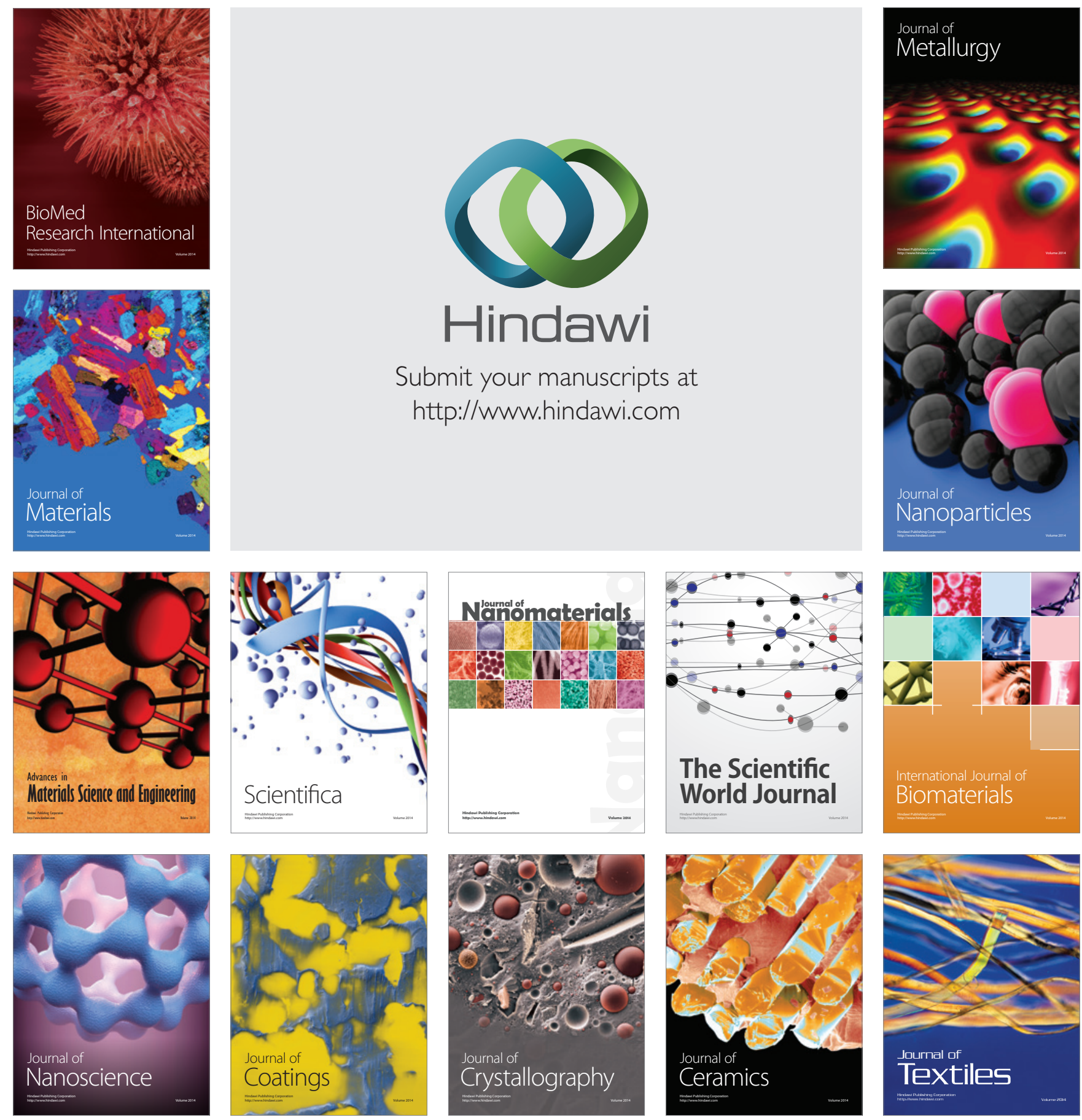